\title{
Ultrasonographic Measurement of Visceral Fat
}

\author{
Tamara Alempijevic, Aleksandra Pavlovic Markovic \\ and Aleksandra Sokic Milutinovic
}

Additional information is available at the end of the chapter

http://dx.doi.org/10.5772/54812

\section{Introduction}

Metabolic syndrome (MS), that has received increased attention in the past few years, consists of multiple, interrelated risk factors of metabolic origin that appear to directly promote the development of atherosclerotic cardiovascular disease (ASCVD). Most important of these underlying risk factors are abdominal obesity and insulin resistance. Other associated conditions include physical inactivity, aging, hormonal imbalance, and genetic or ethnic predisposition ${ }^{1}$.

The measurement of abdominal obesity through waist circumference (WC) has been established as a simple, inexpensive and useful method for the diagnosis of abdominal obesity. Thus, WC has been proposed as a key element for the diagnosis of MS and its use suggested as a part of the routine general physical examination in clinical practice ${ }^{2}$.

Moreover, WC correlates with visceral obesity, and in clinical studies, it has been associated with increased cardiovascular risk ${ }^{3}$. It has been proposed as a part of the routine general physical examination in clinical practice.

It is shown that mesenteric fat thickness is an independent determinant of metabolic syndrome, with an odds ratio of 1.35 for every $1 \mathrm{~mm}$ increase, at least within the observed range of mesenteric fat thickness. The discriminating cut-off point of $10 \mathrm{~mm}$ indicates the presence of metabolic syndrome and identifies subjects with increased intima-media thickness ${ }^{4}$.

Hypotheses relating central adiposity to the metabolic syndrome focus on the newly emerging understanding that adipose tissue (particularly visceral adipose tissue) is a source of factors [including free fatty acids, tumour necrosis factor-alpha (TNF- $\alpha$ )] that impair insulin action in skeletal muscle. In addition, the adipose specific collagen-like molecule, adiponectin, has been found to have antidiabetic, anti-atherosclerotic and anti-inflammatory 
functions. Excessive adipose tissue is associated with a decreased production of adiponectin which may impair insulin sensitivity.

Measurement of mesenteric fat thickness may potentially be developed into an alternative tool to identify subjects at risk for cardiovascular diseases ${ }^{5}$.

Newer clinical studies showed that WC not always correlates with visceral obesity and associated risk for cardiovascular diseases in clinical studies ${ }^{6}$. Therefor, several imaging methods have been proposed for estimation of visceral adipose tissue. Recent advances in imaging techniques and an understanding of differences in the molecular biology of the different adipose tissue depots have been reported. Computed tomography (CT) and especially Magnetic Resonance Imaging (MRI), the gold standard technique, provide non invasive estimation of VAT (visceral adipose tissue) amount safely and accurately. Unfortunately, both MRI and CT are high-cost technologies, and CT requires radiation exposure. In addition, a great variability in the precise definition of adipose tissue compartments by CT and MRI measurements is found in clinical studies. The measurement of visceral fat volume using ultrasonography (US) could calculate as effective as using CT. This method should be practiced in clinics due to its low cost, no side effects and technical suitability ${ }^{7,8}$.

\section{Computed tomography}

In 1999, Yoshizumi et al. presented the standard technique for fat visceral fat measurement at CT which is used in all further published studies ${ }^{9}$. CT (TCT-900S Helix; Toshiba, Tokyo, Japan) was performed with all subjects supine $(120 \mathrm{kV}, 200 \mathrm{~mA}$, section thickness of $5 \mathrm{~mm}$, scanning time of 2 seconds, field of view of $400 \mathrm{~mm}$ ). In subjects, subcutaneous and visceral fat areas were measured on one cross-sectional scan obtained at the umbilicus. A region of interest of the subcutaneous fat layer was defined by tracing its contour on each scan, and the attenuation range of CT numbers (in Hounsfield units) for fat tissue was calculated. A histogram for fat tissue was computed on the basis of mean attenuation plus or minus 2 SD. Intraperitoneal tissue was defined by tracing its contour on the scan; within that region of interest, tissue with attenuation within the mean plus or minus 2 SD was considered to be the visceral fat area. The pixels with attenuation values in the selected attenuation range were depicted as white. From those white regions, the total fat area was calculated by counting the number of pixels in each; the visceral fat area was subtracted, and the remainder was defined as the subcutaneous fat area. Results with this method were compared to those with a computerized planimetric method (KL 4300 Digitizer; Graphtec, Tokyo) and with a fixed attenuation range from 2190 to $230 \mathrm{HU}$ as the standard of reference.

\section{Magnetic Resonance Imaging}

The basic method of visceral fat measurement by MR is as described by Demerath et $\mathrm{al}^{10}$. Images were obtained with a Magnetom Vision 1.5 Tesla whole-body scanner (Siemens, Mississauga, Canada) using a T1-weighted fast-spin echo pulse sequence (TR 322 ms, TE 
$12 \mathrm{~ms})$. The subjects were instructed to lie in the magnet in a supine position with arms extended above the head. A breath-hold sequence ( $\approx 22 \mathrm{~s}$ per acquisition) was used to minimize the effects of respiratory motion on the images. All images were acquired on a 256 $\times 256 \mathrm{~mm}$ matrix and a 480-mm field of view. Slice thickness was $10 \mathrm{~mm}$, and images were obtained every $10 \mathrm{~mm}$ from the 9th thoracic vertebra (T9) to the first sacral vertebra (S1). Depending on the height of the person, this resulted in a total of 21-40 axial images per person. The images were retrieved from the scanner according to a DICOM (Digital Imaging and Communications in Medicine) protocol (National Electrical Manufacturer's Association, Rosslyn, VA). Segmentation of the axial images into VAT and subcutaneous adipose tissue (SAT) areas was performed by 2 trained observers using image analysis software (Slice-OMatic, version 4.2; Tomovision Inc, Montreal, Canada). Tagging of adipose tissue began at the first image, containing the upper margin of the liver and continued down to the L5-S1 image, which increased the likelihood that all intraabdominal adipose tissue was included in the estimate. To calculate VAT and SAT volumes, the VAT and SAT areas for each image were summed across all images. A significant advantage of the contiguous image approach is that no geometrical assumptions have to be made regarding how to interpolate between consecutive slices for the calculation of total volumes.

\section{Ultrasonography}

Ultrasonography (US) is a simple and reliable method for measuring both subcutaneous and visceral fat showing a strong correlation with both adiposities measured with computed tomography scan ${ }^{11}$.

Different techniques are presented by different authors, and no standard method of measurement is still proposed.

In 1990. Armellini et al. ${ }^{12}$ proposed the use of ultrasonography for the first time for the quantification of visceral adiposity as an alternative technique to CT.

There are several proposed techniques, but the most reliable technique of ultrasonographic measurement of fat tissue thickness was presented by Meriño-Ibarra et al. ${ }^{13}$. Sonography measurements were performed using a linear-array probe (Aloka SSD-900, Tokyo, Japan) (7.5 MHz and $42 \mathrm{~mm}$ ) in supine position. It was kept perpendicular to the skin on the upper median abdomen, and longitudinal scan was done in the midpoint between the xiphoid appendix and the navel along the alba line with regard to the surface of the liver, to be almost parallel to the skin. Subcutaneous fat thickness (STh) and area (SA) were measured on the xiphoumbilical line in both longitudinal and transverse views. Measurements were taken 3 times directly from the screen using the electronic calipers at the inner edge of the skin and at the outer edge of the alba line and the fat-muscle interfaces for area. Preperitoneal fat thickness or visceral-fat thickness (VTh) and area (VA) were measured in the same sites and views (Fig. 1). In this case, measurements were taken at the inner edge of the alba line and at the peritoneal line for thickness and area. Then mean values were calculated. Preperitoneal circumference (PC) was calculated as: 


$$
P C=W C-(2 \pi-S T h)
$$

This measurement assumes that WC is a circumference, hence after measuring WC and STh, the intra-abdominal radius and PC can be easily calculated with the formula cited previously. All the subjects were asked to hold their breath during the examination. Special care was taken to keep the probe just touching the skin to prevent compression of the fat layers.

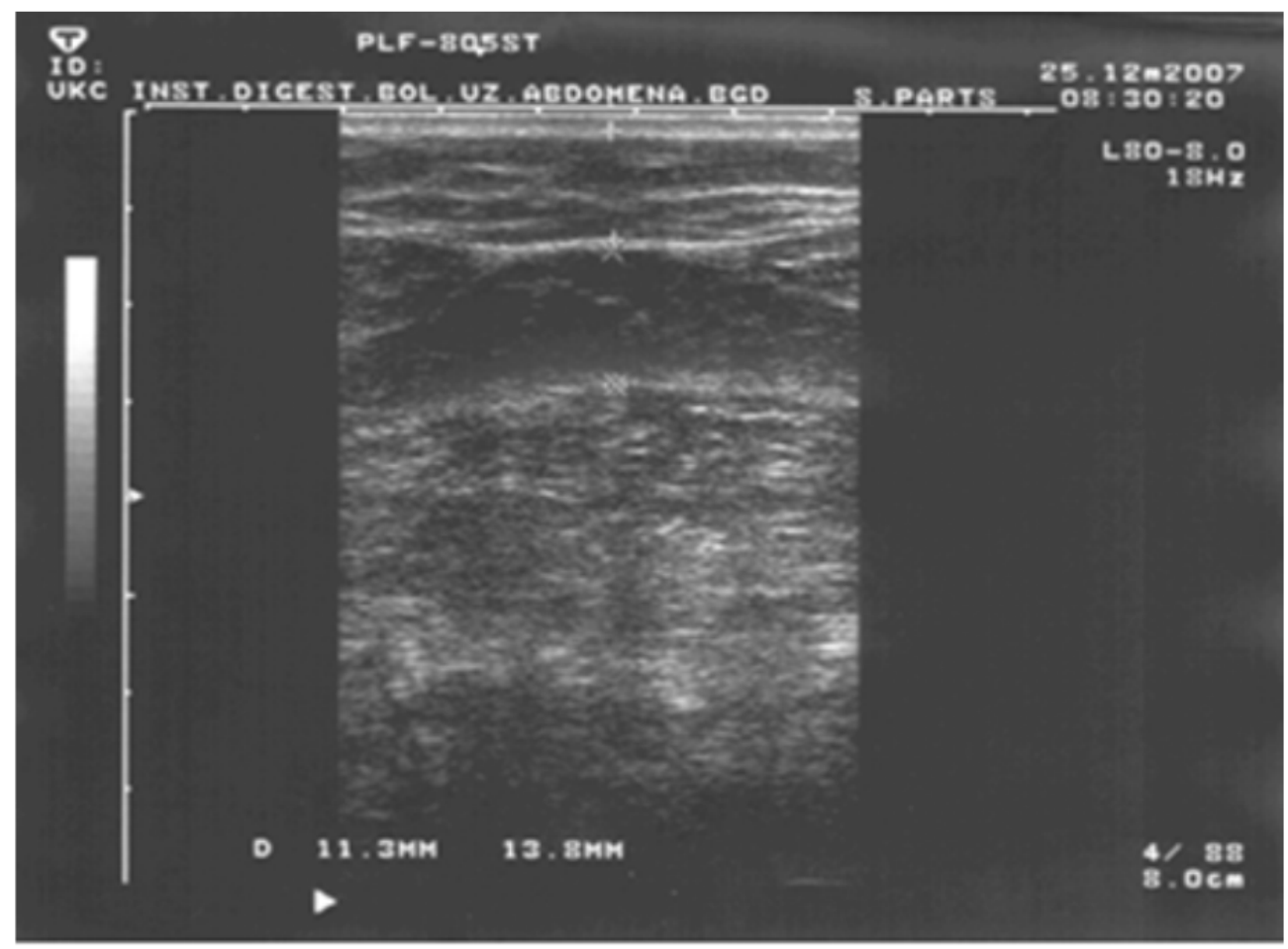

Figure 1. Subcutaneous and visceral fat thickness measurement

Other authors, as well contributed to this topic, Suzuki et al. ${ }^{14}$ proposed a new index as a good non/invasive indicator of viscerl fat deposition. They calculated the abdominal wall fat index as preperitoneal fat/subcutaneous fat ratio. The thickness of subcutaneous as preperitoneal fat layers was measured directly on the screen using electronic callipers. The maximum thickness of preperitoneal fat and the minimum thickness of subcutaneous fat were used as representative markers.

Hirooka et al. ${ }^{15}$ proposed an another technique. This group measured the distance between the internal surface of the abdominal muscle and splenic vein, the distance between the internal surface of the abdominal muscle and the posterior wall of the aorta of the aorta on the umbilicus and the thickness of the posterior right renal wall in the right posterior 
perinephritic space. A $3.5 \mathrm{MHz}$ convex-array probe was used to measure each parameter,other than the thickness of both the subcutaneous and the preperitoneal fat layers at the xyphoid process by performing a longitudinal scan, which was measured using $7.5 \mathrm{MHz}$ linear-array probe. The transducer was placed vertically against the skin as lightly as possible to prevent compression of the fat layers. The distance between the internal surface of the abdominal muscle and the splenic vein was scanned transversely in the midline. When the splenic vein could not be visualized clearly, this vein was detected by using color Doppler flow. The equation was calculated as follows:

$$
\begin{array}{r}
\text { [visceral fat volume }]=-9.008+1.191 \times\left[\begin{array}{l}
\text { distance } \\
\text { between the internal surface of } \\
\text { the abdominal muscle and the } \\
\text { splenic vein }(\mathrm{mm})
\end{array}\right]+ \\
+0.987 \times\left[\begin{array}{l}
\text { distance between the internal } \\
\text { surface of the abdominal muscle and } \\
\text { the posterior wall of the aorta on the } \\
\text { umbilicus }(\mathrm{mm})
\end{array}\right]+ \\
+3.644 \times\left[\begin{array}{l}
\text { thickness of the fat layer of the } \\
\text { posterior right renal wall }(\mathrm{mm})
\end{array}\right]
\end{array}
$$

There was a good correlation between the visceral fat volume calculated by the above equation and the volume by CT described.

Visceral fat amount can be estimated by measurement of perirenal fat, as presented by Kawasaki et al. ${ }^{16}$. This group performed sonography using a Power Vision 8000 scanner (Toshiba, Tokyo, Japan) equipped with a 3.75-MHz convex probe. On sonography, the thickness of combined para- and perirenal fat was measured between the kidney and the inner aspect of the abdominal musculature. Measurements on both sides were averaged. The results correlated with CT measurement, as well as with laboratory values.

\section{Conclusion}

Since 1990, CT has been proposed as the gold standard method to quantify abdominal adiposity ${ }^{17}$. Despre's and Lamarche ${ }^{18}$ observed that a visceral fat area of $130 \mathrm{~cm}^{2}$ was associated with a high risk of cardiovascular events, although prospective studies are still lacking to confirm such an association. In the meantime, other techniques have been used to predict cardiovascular risk. Undoubtedly, BMI is the most common method for estimating body fat, and several epidemiological studies have reinforced its role in the prediction of morbidity and mortality ${ }^{19,20}$. However, an increased BMI does not show which body 
compartment (fat or lean mass) is inadequate and cannot differentiate subcutaneous from visceral fat accumulation. The use of US in the assessment of intra-abdominal fat, initially proposed by Armellini et al. ${ }^{12}$, was further confirmed by strong correlations with the CTdetermined visceral fat area ${ }^{14,21}$. US is expected to be the most specific method because it allows the individual visualization of subcutaneous and intra-abdominal (visceral) fat. In addition, US is a noninvasive and quick method with good reproducibility rates (intraexamination variation $<1 \%$ ) and lower costs than CT scans. However, specific equipment and a welltrained examiner are required. Despite the fact that body composition might be attributable to ethnic differences, changes in the US-determined visceral-to-subcutaneous fat ratio can potentially be monitored for the risk associated with visceral fat accumulation. Also, US may represent a useful method for monitoring weight loss, variations in visceral fat, and associated risks ${ }^{22}$.

Whatever technique is used, ultrasonography is a reliable method for measurement visceral fat amount and assessment of metabolic syndrome presence and risk for cardiovascular diseases development.

\section{Author details}

Tamara Alempijevic, Aleksandra Pavlovic Markovic and Aleksandra Sokic Milutinovic School of Medicine, University of Belgrade, Serbia

Clinic for Gastroenterology and Hepatology, Clinical Center of Serbia, Belgrade, Serbia

\section{References}

[1] Grundy SM, Cleeman JI, MD, Daniels SR, Donato KA, Eckel RH, Franklin BA, et al. Diagnosis and Management of the Metabolic Syndrome. An American Heart Association/National Heart, Lung, and Blood Institute. Scientific Statement. Circulation 2005; 112(17): e285-90.

[2] Wang J. Waist circumference: a simple, inexpensive, and reliable tool that should be included as part of physical examinations in the doctor's office. Am J Clin Nutr 2003; 78(5): 902-3.

[3] Zhu S, Wang Z, Heshka S, Heo M, Faith MS, Heymsfield SB. Waist circumference and obesity-associated risk factors among whites in the third National Health and Nutrition Examination Survey: clinical action thresholds. Am J Clin Nutr 2002; 76(4): 743-9.

[4] Liu KH, Chan YL, Chan WB, Ngor Chan JC, Winnie Chu CW. Mesenteric fat thickness is an independent determinant of metabolic syndrome and identifies subjects with increased carotid intima-media thickness. Diabetes Care 2006; 29(2): 379- 84 .

[5] Mangge H, Almer G, Truschnig-Wilders M, Schmidt A, Gasser R, Fuchs D. Inflammation, adiponectin, obesity and cardiovascular risk. Curr Med Chem. 2010;17(36):4511-20.

[6] Ness-Abramof R, Apovian CM. Waist circumference measurement in clinical practice. Nutr Clin Pract 2008;23(4):397-404. 
[7] Schlett CL, Hoffmann U. [Identification and quantification of fat compartments with CT and MRI and their importance]. Radiologe 2011; 51(5):372-8.

[8] Meng K, Lee CH, Saremi F. Metabolic syndrome and ectopic fat deposition: what can CT and MR provide? Acad Radiol 2010; 17(10):1302-12.

[9] Yoshizumi T, Nakamura T, Yamane M, Islam AHMW, Menju M, Yamasaki K, et al. Abdominal fat: Standardized technique for measurement at CT. Radiology 1999; 211: $283-286$.

[10] Demerath EW, Shen W, Lee M, Choh AC, Czerwinski SA, Siervogel RM, Towne B. Approximation of total visceral adipose tissue with a single magnetic resonance image. Am J Clin Nutr 2007; 85(2): 362-8.

[11] Ribeiro-Filho FF, Faria AN, Kohlmann O Jr, Ajzen S, Ribeiro AB, Zanella MT, et al. Ultrasonography for the evaluation of visceral fat and cardiovascular risk. Hypertension 2001; 38(3 Pt 2): 713-7.

[12] Armellini F, Zamboni M, Rigo L, Todesco T, Bergamo-Andreis IA, Procacci C, et al. The contribution of sonography to the measurement of intra- abdominal fat. J Clin Ultrasound $1990 ; 18(7): 563-7$.

[13] Meriño-Ibarra E, Artieda M, Cenarro A, Goicoechea J, Calvo L, Guallar A, et al. Ultrasonography for the evaluation of visceralfat and the metabolic syndrome. Metabolism 2005; 54(9): 1230-5.

[14] Suzuki R, Watanabe S, Hirai Y, Akiyama K, Nishide T, Matsushima Y, rt al. Abdominal wall fat index, estimated by ultrasonography, for assessment of the ratio of visceral fat to subcutaneous fat in the abdomen. Am J Med 1993 Sep;95(3):309-14.

[15] Hirooka M, Kumagi T, Kurose K, Nakanishi S, Michitaka K, Matsuura B, et al. A technique for the measurement of visceral fat by ultrasonography: comparison of measurements by ultrasonography and computed tomography. Intern Med 2005; 44(8):794-9.

[16] Kawasaki S, Hasegawa O, Satoh S, Numata K, Terauchi Y. Sonographic assessment of fatty liver infiltration using the measurement of para- and perirenal fat thickness. J Clin Ultrasound 2010; 38(9):470-4.

[17] Rossner S, Bo WJ, Hiltbrandt E, et al. Adipose tissue determinations in cadavers-a comparison between cross-sectional planimetry and computed tomography. Int J Obes Relat Metab Disord. 1990;14:893-902.

[18] Despre's JP, Lamarche B. Effects of diet and physical activity on adiposity and body fat distribution: implications for the prevention of cardiovascular disease. Nutr Res Rev. 1993;6: 137-59.

[19] World Health Organization. Obesity: Preventing and Managing the Global Epidemic. Report of a WHO Consulation on Obesity. Geneva, Switzerland; World Health Organization; 1997.

[20] Calle EE, Thun MJ, Petrelli JM, Rodriguez C, Heath CW Jr. Body mass index and mortality in a prospective cohort of US adults. N Engl J Med 1999;341:1097-105.

[21] Tornaghi G, Raiteri R, Pozzato C, et al. Anthropometric or ultrasonic measurements in assessment of visceral fat? A comparative study. Int J Obes Relat Metab Disord 1994;18: 771-5. 
[22] Wirth A, Steinmetz B. Gender differences in changes in subcutaneous and intraabdominal fat during weight reduction: an ultrasound study. Obes Res 1998;6:393-9. 